\title{
Incremento en disponibilidad y control de almacén mediante un análisis del historial de mantenimiento
}

\section{Increase the availability and control of storage through the analysis of the history of Maintenance}

\author{
GONZALEZ-ZARAZUA, Roberto Aldo $\dagger$, SAN MIGUEL-IZA, Sandra Maria*, MARTINEZ- \\ FALCON, Francisco Javier y BELTRAN-GONZALEZ, Elsa
}

Universidad Tecnológica de la Región Centro de Coahuila. Carretera 57 Nte Km 14.5, Tramo Monclova-Sabinas, Monclova Coah. México.

\author{
ID $1^{\text {er }}$ Autor: Roberto Aldo, Gonzalez-Zarazua / ORC ID: 0000-0002-7597-3697, CVU CONACYT ID: 254740 \\ ID 1 ${ }^{\text {er }}$ Coautor: Sandra Maria, San Miguel-Iza / ORC ID: 0000-0002-3012-3250, CVU CONACYT ID: 440841 \\ ID $2^{\text {do }}$ Coautor: Francisco Javier, Martinez-Falcon / ORC ID: 0000-0001-5748-1093, CVU CONACYT ID: 254946 \\ ID $3^{\text {er }}$ Coautor: Elsa, Beltran-Gonzalez / ORC ID: 0000-0002-3987-2071, CVU CONACYT ID: 254564
}

DOI: $10.35429 / J T O .2019 .10 .3 .14 .18$

Recibido 8 de Marzo, 2019, Aceptado, 30 de Junio, 2019

\begin{abstract}
Resumen
La falta de administración del mantenimiento en las empresas trae con sigo una disminución en la disponibilidad de los equipos y altos niveles de inventario que reflejan alguno de los desperdicios. Al realizar un análisis del historial de mantenimiento se observan algunas tendencias que indican algunas desviaciones en el la incidencia de fallas en algunos equipos y el alto consumo o requisición de refacciones o partes para realizar las tareas de mantenimiento. La visión integrada de los conceptos de fiabilidad (capacidad para funcionar continuamente durante un determinado período de tiempo), mantenibilidad (capacidad para ser mantenido preventiva y correctivamente), disponibilidad (capacidad para funcionar en un instante determinado) y el establecimiento de un control de inventario que maneje los niveles óptimos para el aseguramiento del mantenimiento y capacidad productiva de la empresa. Esto permite obtener una programación y ejecución correcta de las actividades de mantenimiento y la planeación del inventario y así aumentar la competitividad de la empresa.
\end{abstract}

Disponibilidad, Inventario, Análisis

\begin{abstract}
The lack of maintenance management in the companies brings with it a decrease in the availability of the equipment and high levels of inventory that affect some of the waste. When performing an analysis of the maintenance history, some trends are observed that indicate some deviations in the incidence of failures in some equipment and the high consumption or requirement of spare parts or parts to perform maintenance tasks. The integrated vision of the concepts of reliability (ability to run continuously for a certain period of time), maintainability (ability to be maintained preventively and correctly), availability (ability to operate at a given time) and the establishment of an inventory control that manages the optimum levels for the maintenance assurance and the productive capacity of the company. This allows to obtain a correct programming and execution of maintenance activities and inventory planning and thus increase the competitiveness of the company.
\end{abstract}

Availability, Inventory, Analysis

Citación: GONZALEZ-ZARAZUA, Roberto Aldo, SAN MIGUEL-IZA, Sandra Maria, MARTINEZ-FALCON, Francisco Javier y BELTRAN-GONZALEZ, Elsa. Incremento en disponibilidad y control de almacén mediante un análisis del historial de mantenimiento. Revista de Operaciones Tecnológicas. 2019. 3-10: 14-18

\footnotetext{
* Correspondencia del Autor (Correo electrónico: sandryiza_16@ hotmail.com)

$\dagger$ Investigador contribuyendo como primer autor.
} 


\section{Introducción}

El enfoque del proyecto en esta empresa del área minera es aumentar la disponibilidad de los equipos y disminuir los niveles y costos de inventario de los componentes y refacciones que se requieren para las actividades de mantenimiento. Dado que un aumento en la disponibilidad de las maquinas lleva consigo un incremento de la productividad y un control de inventarios también mejora los costos de mantenimiento haciendo más rentable la empresa.

\section{Justificación}

La empresa Minera Siderúrgica de Coahuila tiene altos costos de inventario y un deficiente programa de mantenimiento que hacen que el rendimientos de la planta disminuya esto se debe en gran parte a la mala administración que se lleva por la parte del área de mantenimiento, que aunque se cuenta con un historial de este no se tiene un buen seguimiento.

\section{Problema}

Los problemas que se analizaran en el presente proyecto son la falta de seguimiento, planeación del mantenimiento, altos costos de los inventarios de refacciones y partes para el mantenimiento. En la Figura 1. Se observa el historial de mantenimiento.

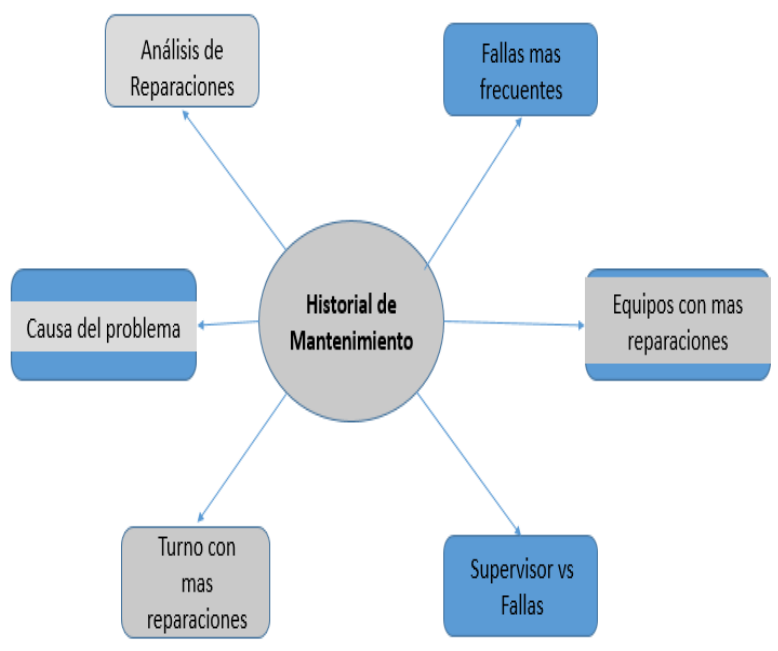

Figura 1 Historial de mantenimiento

\section{Objetivos}

\section{Objetivo General}

Realizar un análisis del historial de mantenimiento a la maquinaria pesada que se utiliza en la mina para determinar las principales fallas y atenderlas de manera rápida y así poder tener un control de inventario en el cual su costo se vea reducido disminuyendo inventarios de refacciones.

\section{Objetivos específicos}

- Realizar un análisis del historial de mantenimiento.

Aumentar la disponibilidad de la máquina.

Determinar las principales refacciones que se utilizan según la frecuencia de mantenimiento.

- Disminuir los costos de inventario.

\section{Marco Teórico}

\section{¿Qué es el mantenimiento?}

Conjunto de técnicas destinadas a conservar equipos e instalaciones en servicio durante el mayor tiempo posible, buscando la más alta disponibilidad y con el máximo rendimiento. [12]. Conjunto de actividades destinadas a mantener o a restablecer un bien a un estado o a unas condiciones dadas de seguridad en el funcionamiento, para cumplir con una función requerida. Estas actividades suponen una combinación de prácticas técnicas, administrativas y de gestión, se pueden observar en la Figura 2.

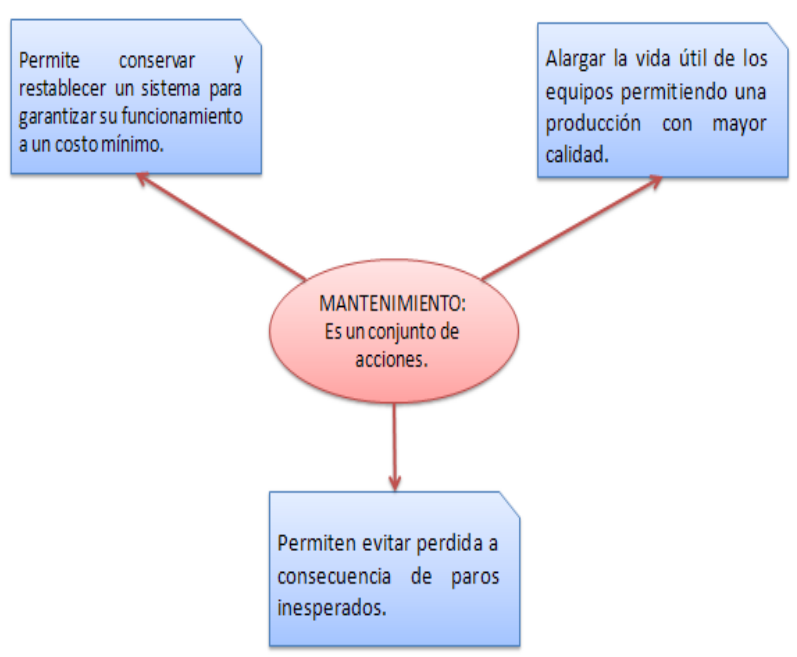

Figura 2 Funciones del mantenimiento 


\section{¿Qué es un historial?}

La recopilación de datos de los equipos tales como marca, modelo, representante, posible proveedor nos facilita la tarea de llevar un mejor control del mantenimiento.

La documentación de las fallas, sus soluciones y refacciones utilizadas nos permite en caso de que se repita resolverlo con mayor rapidez y deducir los métodos de prevención necesarios para evitar que vuelva a suceder. En el caso de que se tenga que hacer alguna modificación al equipo aquí también se documenta la forma en que se realizó.

Datos que puede contener un historial

Datos generales. Nombre del equipo, Marca, Serie, Modelo, Representante, Capacidad de diseño, observaciones generales, imagen del equipo.

Componentes principales. Por facilidad dividimos el equipo en componentes eléctricos, mecánicos, etc. después una subdivisión como motor principal, arrancador, bombas, etc.

Lista de refacciones. Refacciones que consideramos críticas y su cantidad en almacén, así como posible proveedor.

Historia. Anotación de fallas, Trabajos, Modificaciones, etc. anotando Fecha, Falla o trabajo, Solución, si es correctivo o Preventivo, Tiempo, Refacciones utilizadas y encargado.

\section{Disponibilidad}

La disponibilidad propiamente dicha es el cociente entre el tiempo disponible para producir y el tiempo total de parada. Para calcularlo, es necesario obtener el tiempo disponible, como resta entre el tiempo total, el tiempo por paradas de mantenimiento programado y el tiempo por parada no programada. Una vez obtenido se divide el resultado entre el tiempo total del periodo considerado.

Las horas de parada por mantenimiento que deben computarse son tanto las horas debidas a paradas originadas por mantenimiento programado como el no programado.

\section{Inventarios}

El inventario es una herramienta básica para que las empresas puedan gestionar las necesidades de cada una de las existencias o productos, cuando realizar el pedido al proveedor y la cantidad necesaria. [3]

El inventario se crea cuando el volumen de materiales, partes o bienes terminados que se recibe es mayor que el volumen de los mismos que se distribuye. [4] El Sistema de Inventario Perpetuó es el mejor sistema de registro de mercancías para cualquier empresa debido a su buena organización. También es conocido como Sistema de Inventario Constante o Permanente, este organiza la información de tal manera que pueda ser localizada en cualquier momento, sin problemas y por quien lo necesite, del valor del inventario final, las salidas de los consumibles dentro de la empresa, el total de las compras de dichos consumibles. [5]

Definición de Almacén:

Es un lugar o espacio físico para el almacenaje de bienes dentro de la cadena de suministro. [6] Los almacenes son una infraestructura imprescindible para la actividad de todo tipo de agentes; El Sistema de Inventarios Constantes o Perpetuos, es el más completo de los sistemas para el control de las operaciones con mercancías, y presenta como ventaja el control "constante" de los costos de la mercancía comprada y de las correlativas salidas de almacén.

El procedimiento de Inventarios perpetuos, consiste en registrar las operaciones de mercancías de tal manera que se pueda conocer en cualquier momento el valor del inventario final, las salidas de los consumibles dentro de la empresa, el total de las compras de dichos consumibles.

Para tener este control de inventario perpetuo se diseñó en Excel una serie de tablas para tener una base de datos donde indique los artículos y mercancía comprada que entra al almacén de igual manera de los artículos de salida del mismo (que en este caso los denominamos consumibles). 


\section{Metodología de Investigación}

En Minera Siderúrgica de Coahuila S.A. de C.V. se cuenta con una amplia área de oportunidad, ya que únicamente se realizan reportes los cuales no tienen organizados y son ineficientes. Además, no se cuenta con la manera de analizar que reparaciones son las que realizan generalmente $o$ que fallas son las más comunes en los equipos. Por lo cual, se desarrolló este proyecto.

El proyecto se sustentó, por medio de una base de datos que tuviera como función ser un Historial de Mantenimiento para el registro de fallas y/o reparaciones en los equipos $\mathrm{y}$ maquinaria de la mina. El análisis causa-raíz se observa en la Tabla 1.

Deficiencia en el control de reparaciones (incidencias) y ejecución del mantenimiento en la máquina y/o equipo pesado.

\begin{tabular}{|c|c|}
\hline Materias primas & $\begin{array}{l}\text { - Falta de control de inventario. } \\
\text { - Falta de repuestos en el taller y } \\
\text { almacén por falta de un control. }\end{array}$ \\
\hline Maquina & $\begin{array}{l}\text { - Falta de mantenimiento. } \\
\text { - Máquinas obsoletas. } \\
\text { - No hay control de reparaciones } \\
\text { y/o fallas. }\end{array}$ \\
\hline Medio ambiente & $\begin{array}{l}\text { - Condiciones del clima } \\
\text { (Lluvias). } \\
\text { - Deficiencia en la iluminación } \\
\text { en áreas de trabajo. }\end{array}$ \\
\hline Mano de obra & $\begin{array}{l}\text { - Falta de capacitación. } \\
\text { - Mal uso de recursos. } \\
\text { - Mal uso de herramientas. } \\
\text { - Planificación inadecuada de } \\
\text { mantenimiento }\end{array}$ \\
\hline Metodo & $\begin{array}{l}\text { - Control de documentos } \\
\text { deficiente. } \\
\text { - Mal control de los registros. } \\
\text { - Falta de seguimiento de los } \\
\text { planes de mantenimiento } \\
\text { preventivo. }\end{array}$ \\
\hline
\end{tabular}

Tabla 1 Análisis causa-raíz

Actualmente en almacén, el inventario se lleva a cabo registrando salidas y entradas en una bitácora, posteriormente realizando un conteo de los artículos existentes, para al final comprar el resultado obtenido del conteo de las bitácoras, la empresa lleva a cabo el inventario cada 30 días. Con el fin de tener un mejor control acerca de los artículos que entran al almacén, se realizó este proyecto, para que la empresa cuente con un sistema computarizado y sea más rápido el chequeo de la realización de inventarios, y se tenga un mejor control del almacén.

Mediante una lluvia de ideas se analizó si se acepta la implementación del Historial de Mantenimiento.
Se puede analizar con tablas dinámicas diferentes análisis, como son:

- $\quad$ Reparaciones más frecuentes.

- $\quad$ Equipos con más reparaciones.

- $\quad$ Turno con más reparaciones y fallas.

- Refacciones o partes más dañadas.

Además de tener un control en la planeación de mantenimiento y un registro de las reparaciones y/o fallas que se realizan en cada uno de los equipos pesados.

\section{Resultados y conclusiones}

Con la aceptación de la implementación del Historial de Mantenimiento, la empresa ahora cuenta con un sistema computarizado fácil y rápido, para así capturar las reparaciones y/o fallas de los equipos. Con lo cual se obtuvieron los siguientes resultados:

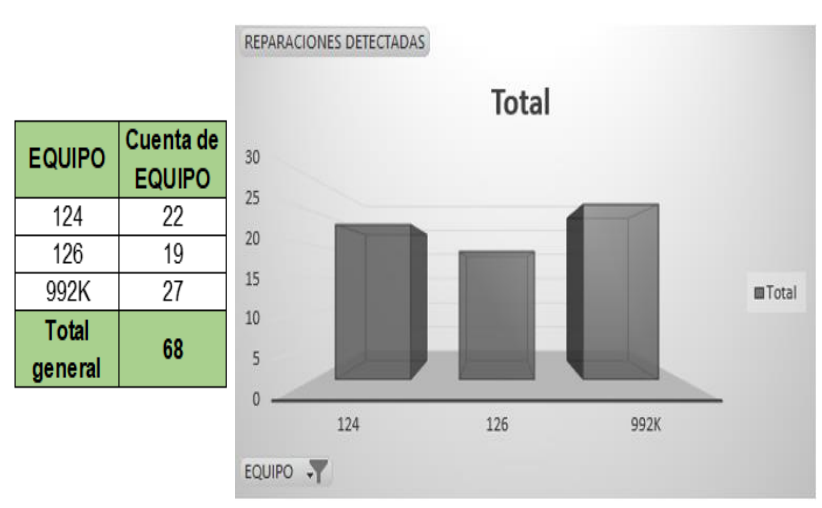

Figura 4 Reparaciones más frecuentes

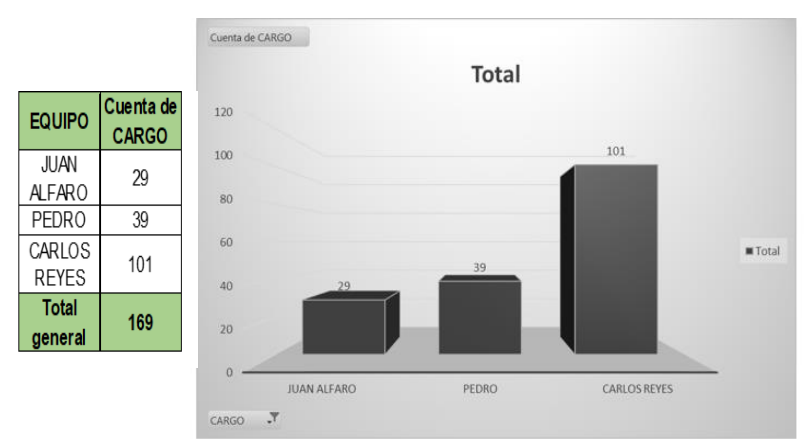

Figura 5 Supervisores que detectaron más fallas

GONZALEZ-ZARAZUA, Roberto Aldo, SAN MIGUEL-IZA, Sandra Maria, MARTINEZ-FALCON, Francisco Javier y BELTRANGONZALEZ, Elsa. Incremento en disponibilidad y control de almacén mediante un análisis del historial de mantenimiento. Revista de Operaciones Tecnológicas. 2019 


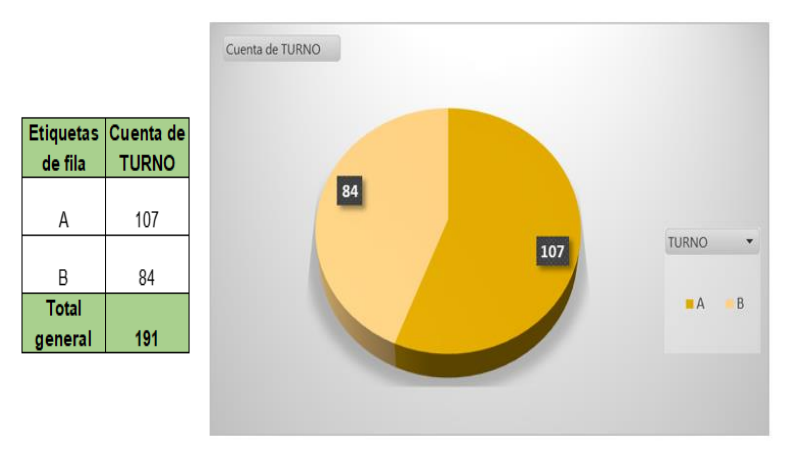

Figura 6 Detección de fallas por turno

A lo largo del desarrollo de este proyecto, se analizó las entradas de artículos y consumibles mediante facturas, remisiones, notas de compra, etc. También las salidas de dichos consumibles del almacén a través de comprobantes de salida que le denominamos vales de almacén. Derivado de la implementación del Sistema integral de control de inventarios.

Se han detectado los siguientes puntos:

- $\quad$ Implementación de vales de almacén.

- $\quad$ Optimización de la compra de artículos.

- $\quad$ Disminución de compras en un 8\%

- Implementación de un cargo para cada artículo que entra y/o sale del almacén, ya sea un departamento y/o equipo.

Eficaz y rápido sistema de inventario, ya que la empresa realiza Inventarios perpetuos o continuos.

Realización de inventarios semanales, mensuales, trimestrales, semestrales, anuales etc.

Tener la información necesaria disponible para realizar una rendición de cuentas de las entradas y salidas, para el análisis y posterior toma de decisiones para mejorar los costos.

\section{Referencias}

[1] Santiago García Garrido. (2010). La contratación del mantenimiento industrial. Madrid: Díaz de Santos.

[2] Santiago García Garrido. (2003). Organización y gestión integral de mantenimiento. Madrid: Díaz de Santos.

[3] Antonia Cruz Fernández. (2017). Gestión de inventarios. Málaga: IC Editorial Innovación y Cualificación.
[4] Lee J. Krajewski, Larry P. Ritzman. (2000). Administración de operaciones: Estrategia y análisis. Naucalpan de Juárez, México: Pearson.

[5] Charles T. Horngren, Gary L. Sundem, John A. Elliott. (2000). Introducción a la Contabilidad Financiera. México: Pearson.

[6] (2003). Técnicas Administrativas de Compra-Venta. Málaga: IC Editorial Innovación y Cualificación. 